\title{
Effects of Vitamin K-1 and Menaquinone-7 on Vascular Function and Blood Pressure in Warfarin-Induced Calcification-Model
} in Rats

\author{
Aino Siltari' ${ }^{1}$ Niko Wickholm¹, Anne S. Kivimäki' ${ }^{1}$, Kaisa Olli², Krista Salli², \\ Kirsti Tiihonen' ${ }^{2}$, Riitta Korpela' ${ }^{1}$, Heikki Vapaatalo' ${ }^{1}$ \\ ${ }^{1}$ Institute of Biomedicine, Pharmacology, University of Helsinki, Helsinki, Finland \\ ${ }^{2}$ DuPont Nutrition and Health, Kantvik Active Nutrition, Kantvik, Finland \\ Email: aino.siltari@helsinki.fi
}

Received 24 September 2014; revised 15 October 2014; accepted 30 October 2014

Academic Editor: Shengyong Yang, Sichuan University, China

Copyright (C) 2014 by authors and Scientific Research Publishing Inc.

This work is licensed under the Creative Commons Attribution International License (CC BY).

http://creativecommons.org/licenses/by/4.0/

(c) (i) Open Access

\section{Abstract}

Given that vascular calcification is inversely correlated with the clinical intake of menaquinone, a rat model of warfarin-induced calcification may be useful for testing menaquinone and vitamin K-1 potential effects on vascular function. The aim of the present study was to investigate effects of vitamin K-1 and menaquinone-7 treatments on blood pressure and vascular function in warfarininduced vascular calcification model during five-week intervention in normotensive Wistar-Kyoto rats. Blood pressure was measured weekly, and at the end of the intervention in vitro vascular reactivity measurements were done. Alizarin Red $S$ and von Kossa stainings were used to record possible calcification of aortic sections. Routine clinical chemistry was done from serum and urine samples. Vascular calcification was seen only in a few warfarin-treated animals in histological staining. Warfarin-treatment did not change significantly blood pressure of the rats. Warfarintreatment increased slightly the endothelium-dependent relaxation of aorta after the L-type calcium channels were blocked. Also the vascular relaxation improved after NOS inhibition in the aorta of the healthy controls and menaquinone- 7 treated animals, indicating that the relaxation in those groups was not totally dependent on NO. Clinical chemistry from serum showed some differences in urea, creatinine as well as lipid and glucose metabolism between the healthy controls and warfarin-treated rats. 


\section{Keywords}

\section{Vascular Calcification, Vitamin K-1, Menaquinone-7, Blood Pressure, Vascular Function}

\section{Introduction}

Vascular calcification, the extracellular deposition of calcium in the vascular wall, occurs in relation to a number of vascular disorders, including vascular smooth muscle apoptosis and endothelial dysfunction. It may also lead to more severe cardiovascular diseases like hypertension and atherosclerosis [1].

Vascular calcification is inversely correlated with vitamin K-2 (menaquinone, MK) intake in humans [2]-[4]. Therefore, warfarin-induced calcification in rats may offer a potential model for testing the possible actions of vitamin K-1 (K-1) and MK on vascular function.

Several weeks of warfarin treatment has been shown to induce artery wall (elastic lamellae) and heart valves calcification [5]-[12] in experimental models. This phenomenon is not related to anticoagulation or decreased activity of factors II, VII, IX, X, or protein C and S. In addition to these vitamin K-dependent proteins produced in the liver, there are a number of extra-hepatic vitamin K-dependent proteins including matrix Gla protein (MGP), a 10-kDA protein that contains five residues of the vitamin K-dependent calcium binding amino acids, gamma-carboxylglutamic acid (Gla). Other vitamin K-dependent proteins include osteocalcin, nephrocalcin, plaque Gla protein, growth arrest specific gene 6 (Gas 6) and proline rich Gla proteins [13]. Reduced carboxylation of these proteins is likely to be an unavoidable side-effect of warfarin therapy. MGP knockout mice develop severe calcification of the arterial system suggesting that MGP normally prevents this process [14]. Warfarin seems to inhibit the activation of MGP and therefore causes vascular calcification in animal models [15] [16].

The aim of the present study was to investigate the effects of K-1 and menaquinone-7 (MK-7) on vascular calcification in an animal model and to test the suitability of the warfarin model for this purpose. Blood pressure, vascular function and basic clinical chemistry monitoring widened our knowledge on the long-term effects of the warfarin alone or together with vitamin K-1 or MK-7 treatments.

\section{Materials and Methods}

\subsection{Diets and Animals}

Male Wistar-Kyoto rats (WKY) (10 weeks old, weight 242 - 245 g, purchased from Scanbur Ab, Sollentuna, Sweden), with free access to tap water and irradiated (0.9 Mrad) vitamin K-deficient diet (Harlan, Venray, The Netherlands), were randomized based on systolic blood pressure value and weight into four groups of 10 animals (housed five in a gage) in a standard experimental laboratory (illuminated from 7 am to 7 pm, humidity $55 \% \pm 15 \%$, temperature $22^{\circ} \mathrm{C} \pm 2{ }^{\circ} \mathrm{C}$ ). The protocol was approved by National Animal Experimentation Committee of Finland according to EC Directive 86/609/ECC and Finnish Experimental Animal Act 62/2006.

The warfarin-induced calcification model and diets were used as reported earlier [7] [8]:

Groups were as follows: Healthy control group (H-C) received vitamin K-deficient diet containing K-1 (Merck, Darmstadt, Germany) $5 \mu \mathrm{g} / \mathrm{g}$ feed (which is normal K-1 level in a standard rodent diet in Harlans diets). Warfarin control group (W-C) received $3 \mathrm{mg}$ of warfarin/g feed and $1.5 \mathrm{mg} \mathrm{K}-1 / \mathrm{g}$ feed. Vitamin K-1 group (K-1) received $3 \mathrm{mg}$ of warfarin/g feed $1.5 \mathrm{mg} \mathrm{K}-1 / \mathrm{g}$ feed with simultaneous administration of K-1 (in total $2-3 \mathrm{mg} / \mathrm{g}$ feed). Menaquinone-7 group (MK-7) received $3 \mathrm{mg}$ of warfarin/g feed and $1.5 \mathrm{mg} \mathrm{K}-1 / \mathrm{g}$ feed with simultaneous administration of MK-7 (100 $\mu \mathrm{g} / \mathrm{g}$ feed) (generous gift from Kappa Bioscience, Oslo, Norway) (Table 1). The rat strain, sex and age as well as the warfarin doses and treatment period were based on previous studies [5]-[8].

The intervention lasted for 5 weeks and consumption of feed and water were recorded daily. K-1 and MK-7 were diluted in corn oil and warfarin (Sigma-Aldrich, St. Louis, MO, USA) was mixed directly into the feed powder. All diets contained equivalent amount of corn oil (7 ml/kg feed). K-1 and MK-7 are sensitive to light, therefore all diets were prepared and stored as dark as possible. High dose ( $3 \mathrm{mg} \mathrm{K}-1 / \mathrm{g}$ feed) of vitamin K-1 (K-1 group) showed toxic effects in two weeks, but the animals recovered after reduction of the dose (2 mg vitamin K-1/g diet). 
Table 1. Vitamin K-1, warfarin and menaquinone-7 (MK-7) content in the feed (mg/g feed) and daily intake of vitamin K-1, warfarin, and MK-7 (calculated per rat) during the five-week study-period. Mean \pm SEM, $\mathrm{n}=10$.

\begin{tabular}{|c|c|c|c|}
\hline & \multicolumn{3}{|c|}{ Content per gram feed (mg/g): } \\
\hline & Vitamin K-1 & Warfarin & MK-7 \\
\hline Healthy control & 0.01 & - & - \\
\hline Vitamin K-1 group & $2.0-3.0$ & 3.00 & - \\
\hline Menaquinone-7 group & 1.50 & 3.00 & 0.10 \\
\hline \multirow[t]{3}{*}{ Warfarin control } & 1.50 & 3.00 & - \\
\hline & \multicolumn{3}{|c|}{ Daily intake (mg/rat): } \\
\hline & Vitamin K-1 & Warfarin & MK-7 \\
\hline Healthy control & $0.16 \pm 0.01$ & - & - \\
\hline Vitamin K-1 group & $70.9 \pm 9.4$ & $92.2 \pm 6.0$ & - \\
\hline Menaquinone-7 group & $43.5 \pm 1.4$ & $87.0 \pm 2.8$ & $2.91 \pm 0.1$ \\
\hline Warfarin control & $49.2 \pm 1.7$ & $98.4 \pm 3.5$ & - \\
\hline
\end{tabular}

\subsection{Blood Pressure Measurements}

Blood pressure and heart rate measurements (CODA-program, Kent Scientific Corporation, Torrington, CT, USA) were carried out weekly by an automatic tail-cuff method by the same person (AS) at the same time of day. Rats were pre-warmed at $32^{\circ} \mathrm{C}$ for 10 - 15 min before measurements. Blood pressure and heart rate measurement were repeated 20 times (each rat) and systolic blood pressure (SBP), diastolic blood pressure (DBP) and heart rate were calculated as the mean of the measurements accepted by the CODA program (at least 5 accepted measurements).

\subsection{Sample Collection}

After 5 weeks, rats were killed with isoflurane gas (5\%) and blood was collected by heart puncture and placed in clot Act z containing and silicon coated tubes (Venosafe, Terumo, Lueven, Belgium), centrifuged at $4^{\circ} \mathrm{C}$ and $3000 \mathrm{~g}$ for $10 \mathrm{~min}$ after serum was collected, and kept at $-80^{\circ} \mathrm{C}$ until further analysis. Urine samples were collected from bladder puncture and kept at $-80^{\circ} \mathrm{C}$. Weights of the heart, left ventricle, and left kidney were measured. Parts of the descending aorta were fixed in neutral buffered $10 \%$ formaldehyde (Sigma-Aldrich) and after $48 \mathrm{~h}$ placed in $70 \%$ ethanol and embedded in paraffin for further histological studies. Mesenteric arteries and the lower part of the descending aorta was placed in pre-oxygenated $\left(\mathrm{O}_{2} / \mathrm{CO}_{2} 95 \% / 5 \%\right.$, AGA, Riihimäki, Finland) ice-cold Krebs buffer (pH 7.4 - 7.6, composition in mM: $\mathrm{NaCl} 119.0, \mathrm{NaHCO}_{3}$ 25.0, glucose 11.1, KCl 4.7, $\mathrm{CaCl}_{2}$ 1.6, $\mathrm{KH}_{2} \mathrm{PO}_{4}$ 1.2, $\mathrm{MgSO}_{4}$ 1.2).

\subsection{Vascular Reactivity Studies}

Changes in vascular tone and endothelial function were studied in standard organ bath chambers with a computerized system (EMKA Technologies, Paris, France). Endothelium-intact mesenteric artery rings and descending aorta rings without adherent connecting tissue were hooked with stainless steel hooks (diameter $0.1 \mathrm{~mm}$ for mesenteric arteries and $0.2 \mathrm{~mm}$ for aortas), placed in oxygenated Krebs buffer in organ bath chambers (10 ml, $37^{\circ} \mathrm{C}$ ), and vascular reactivity was studied using vasoconstricting and vasodilating drugs.

The vascular reactivity study protocol was as follows: After $1 \mathrm{~h}$ equilibration time in $1.5 \mathrm{~g}$ baseline tone, about $3 \mathrm{~mm}$ long rings were contracted using $60 \mathrm{mM} \mathrm{KCl}$ to initiate a contraction response. Intact endothelial function was tested using $10 \mu \mathrm{M}$ acetylcholine (ACh) after $1 \mu \mathrm{M}$ phenylephrine (PE) contraction. Cumulatively ACh (1 nM - $10 \mu \mathrm{M})$ relaxation and endothelium-independent relaxation with sodium nitroprusside (SNP) (1 $\mathrm{nM}-1 \mu \mathrm{M}$ ) were tested. The role of calcium channels and nitric oxide (NO) were studied using 15 min preincubation of $1 \mu \mathrm{M}$ nifedipine (L-type calcium channel blocker) or $100 \mu \mathrm{M} \mathrm{L}-\mathrm{N}^{\mathrm{G}}$-Nitroarginine methyl ester (L-NAME, nitric oxide synthase inhibitor) before PE contraction and cumulative ACh relaxation. The rings were washed three times with Krebs buffer and equilibrated for 20 - 30 min between the different measurements. The concentrations reported here are the final concentrations in the organ chamber. All compounds were pur- 
chased from Sigma-Aldrich.

\subsection{Clinical Chemistry Measurements}

Routine clinical chemistry (ADVIA 1650 Chemistry System, Siemens Healthcare Diagnostics Inc., Deerfield, IL, USA) was used to detect electrolytes $\left(\mathrm{K}^{+}, \mathrm{Na}^{+}, \mathrm{Cl}^{-}\right.$, and $\left.\mathrm{Ca}^{2+}\right)$, albumin, glucose, urea, total protein, inorganic phosphate, and creatinine from urine and serum. Also, alanine aminotransferase, alkaline phosphatase, aspartate transaminase, creatine kinase, total bilirubin, high-density lipoprotein (HDL), low-density lipoprotein (LDL), triglycerides, and total cholesterol were measured from serum using the same multichannel analyser.

\subsection{Blood Coagulation Measurements}

Prothrombin time (INR, international normalized ratio) was determined using the standardized technique (GoaguChek XS System-instrument and Guagu Chek XS PT Test, Roche Diagnostic, Mannheim, Germany).

\subsection{Histological Staining}

Possible aortic calcification was evaluated from histology samples using Alizarin Red S and von Kossa stainings. In von Kossa staining, nuclear fast red (Merck, Darmstadt, Germany) was used as a counterstain. Paraffin-embedded samples samples ( $n=6-8$ each group) were cut longitudinally into serial sections of $6-7 \mu \mathrm{m}$ with microtome (several sections per sample). After deparaffinization and hydration the tissue sections were stained using 1.1\% Alizarin Red S solution (AppliChem, Kongens Lyngby, Denmark) (pH 4.2 adjusted with 0.5\% ammonium hydroxide) or with $5 \%$ silver nitrate solution (Sigma-Aldrich) (1 hour incubation in bright light). After dehydration, the samples were mounted with DePex mounting medium (VWR international, Leicestershire, England) and analyzed by light microscope (Leica Microsystems, Wetzlar, Germany).

\subsection{Statistical Analyses}

All the measurements and statistical evaluations were performed blinded. The results are presented as mean \pm SEM. Statistical analyses were performed using GraphPad Prism software (version 4.02). One way analysis of variances (ANOVA) followed Tukey multiple comparison tests were used to compare different groups. Difference was considered significant if p-value $<0.05$.

\section{Results}

\subsection{Body and Organ Weights, Feed and Water Consumption}

Table 1 illustrates the daily dosing of feed, water, vitamin K-1, warfarin and MK-7 in different groups during 5 weeks' study period. Warfarin-treatment influenced the development of body weight.

The healthy control group $(\mathrm{H}-\mathrm{C})$ was heavier than the other groups $(\mathrm{p}<0.01)$ and thus, kidney, heart and left ventricle (all related to body weight) were smaller compared to the warfarin-treated groups (Table 2) $(\mathrm{p}<0.01$ for kidney). There were no differences in feed consumption between the groups, thus, the higher body weight of the healthy group cannot be explained by that (Table 2). Warfarin-treated rats consumed more water than the healthy controls $(\mathrm{p}<0.01)$. There were no significant differences in fluid consumption between three warfarin consumed groups (Table 2).

\subsection{Blood Pressure and Heart Rate}

There were no differences in systolic or diastolic blood pressure or heart rate of the rats during the intervention. Different baseline values were taken into account in statistical tests. Warfarin-treatment did not influence blood pressure or heart rate (Figure 1).

\subsection{Prothrombin Time}

Warfarin-treatment did not influence the blood coagulation process, INR values were at the same levels in the beginning and at the end of the intervention (Figure 2), indicating that vitamin K-1 balanced warfarin anticoagulation effects, and the rats did not suffer bleeding during the intervention. 
Table 2. Daily water (ml) and feed (g) consumption per rat and body mass (g) and the weight of kidney, heart, and left ventricle related to body mass $(\mathrm{mg} / \mathrm{g})$ of the rats. Mean \pm SEM, $n=9-10$.

\begin{tabular}{cccc}
\hline & Water & Feed & Body mass \\
\hline Healthy control & $17 \pm 1^{*}$ & $32 \pm 1$ & $398 \pm 6^{*}$ \\
Vitamin K-1 group & $25 \pm 1$ & $31 \pm 2$ & $320 \pm 7$ \\
Menaquinone-7 group & $24 \pm 2$ & $29 \pm 1$ & $324 \pm 4$ \\
Warfarin control & $24 \pm 1$ & $33 \pm 1$ & $341 \pm 6$ \\
\hline Healthy control & Kidney & Heart & Left ventricle \\
Vitamin K-1 group & $3.37 \pm 0.05^{*}$ & $2.58 \pm 0.04$ & $2.03 \pm 0.03$ \\
Menaquinone-7 group & $3.66 \pm 0.05$ & $2.71 \pm 0.03$ & $2.12 \pm 0.04$ \\
Warfarin control & $3.70 \pm 0.08$ & $2.70 \pm 0.04$ & $2.07 \pm 0.04$ \\
\hline
\end{tabular}

"Healthy vs. others $\mathrm{p}<0.01$.
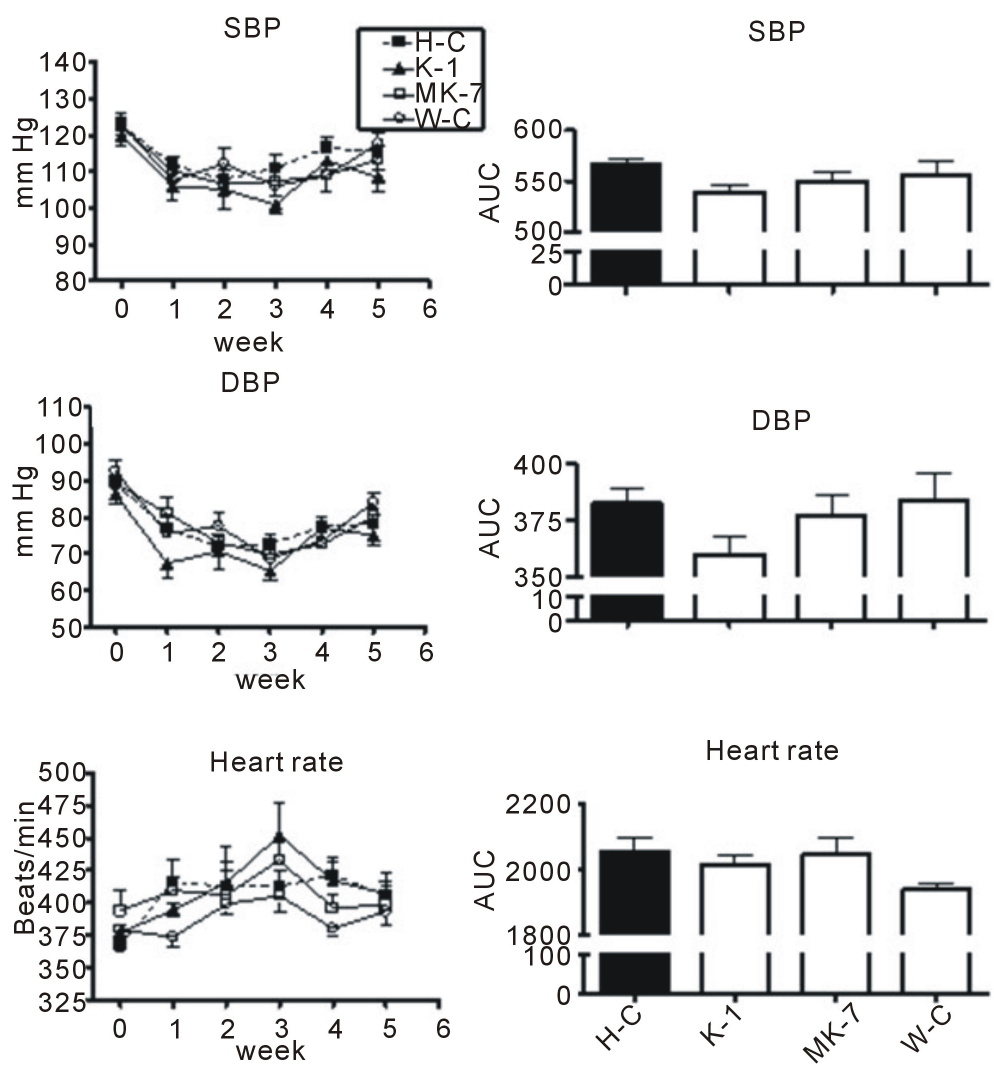

Figure 1. Systolic blood pressure (SBP), diastolic blood pressure (DBP), and heart rate during the five-week intervention with warfarin and vitamin-K derivatives and area under curve (AUC) calculated from the data of the left panel. $\mathrm{H}-\mathrm{C}=$ Healthy control, $\mathrm{K}-1$ = Vitamin K-1, MK-7 = Menaquinone-7 and $\mathrm{W}-\mathrm{C}=$ Warfarin control. Mean $\pm \mathrm{SEM}, \mathrm{n}=10$ in each group.

\subsection{Vascular Function}

Vascular function was measured using endothelium-intact rings of mesenteric artery (small vessel) and aorta (large vessel).

Potassium chloride-induced $(60 \mathrm{mM}, \mathrm{KCl})$ contraction in mesenteric arteries was more pronounced in the healthy group (H-C) and a similar tendency was seen in phenylephrine (PE) contraction (Figure 3). There were no differences in aorta contraction between the groups. 


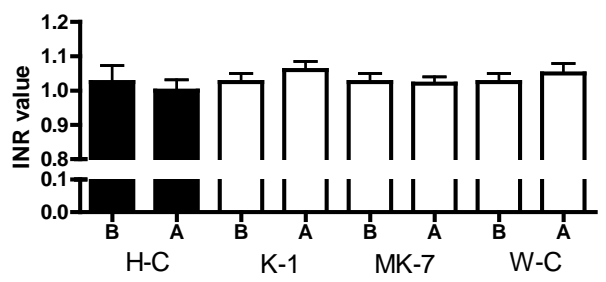

Figure 2. INR values before $(\mathrm{B})$ and after $(\mathrm{A})$ the intervention. $\mathrm{H}-\mathrm{C}=$ Healthy control, K-1 = Vitamin K-1, MK-7 = Menaquinone-7, W-C = Warfarin control. Mean \pm SEM, $n=5$ in each group.

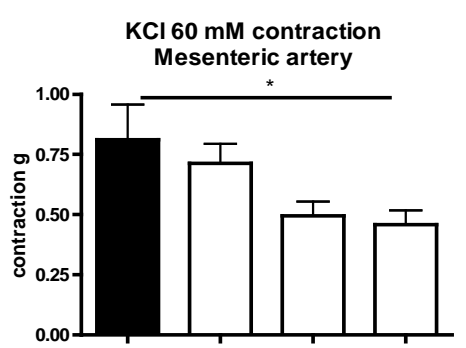

PE contraction Mesenteric artery

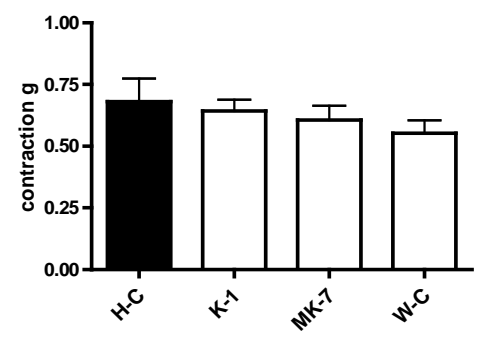

$\mathrm{KCl} 60 \mathrm{mM}$ contraction

$$
\text { Aorta }
$$

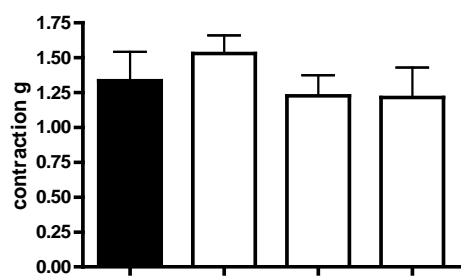

PE contraction Aorta

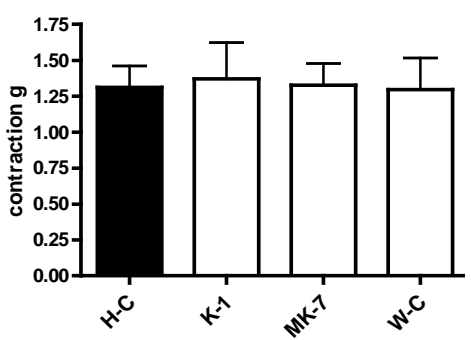

Figure 3. KCl- and phenylephrine (PE)-induced contractions in mesenteric arteries and in aortas after five-week intervention. $\mathrm{H}-\mathrm{C}=$ Healthy control, $\mathrm{K}-1=$ Vitamin K-1, MK-7 = Menaquinone-7 and W-C = Warfarin control. Mean \pm SEM, $\mathrm{n}=7-10$. $^{*} \mathrm{p}<0.05$.

In Figure 4, the endothelium-dependent and -independent relaxations in mesenteric arteries and aorta are shown. Endothelium-dependent vasorelaxation was recorded using acetylcholine (ACh) cumulatively. In the warfarin control group (W-C) relaxation was slightly reduced. In aortas of the healthy group (H-C) relaxation was the smallest (Figure 4(a)), however, the difference was not statistically significant. There were no differences in sodium nitroprusside (SNP)-induced endothelium-independent vasorelaxation (Figure 4(b)), indicating normal smooth muscle function. L-type calcium-channels were blocked using nifepidine (Figure 4(c)). In mesenteric arteries there were no differences between the groups. The best relaxation was seen in the aortas of warfarin control group (W-C) and the worst in the healthy controls (H-C).The relaxation of MK-7 and K-1 groups was between the W-C and $\mathrm{H}-\mathrm{C}$ groups ( $\mathrm{H}-\mathrm{C}$ vs. other groups $\mathrm{p}<0.05$ ) (Figure 4(c)).

Inhibition of nitric oxide synthatase (NOS) with L-NAME in mesenteric artery relaxation reduced similarly ACh relaxation in all treatment groups (Figure 4(d)). The blockade was not total leaving about 25\% relaxation obviously related to endothelial prostacyclin and endothelium-derived hyperpolarizing factor (EDHF) production. In aortas, the healthy controls and the MK-7 group showed some relaxation even after NOS inhibition, while in the two other groups no relaxation to ACh was seen, indicating nitric oxide's main role in endothelium dependent relaxation (H-C vs. vitamin K-1 p < 0.01) (Figure 4(d)).

\subsection{Vascular Calcification}

Vascular calcification was measured in histological samples of descending aorta using Alizarin Red S and von 
Mesenteric artery

(a)
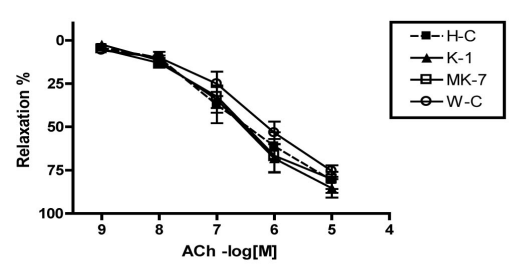

(b)

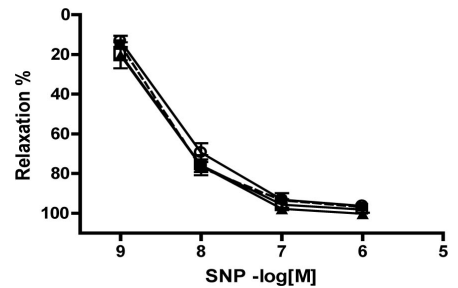

(c)

Nifepidine incubation

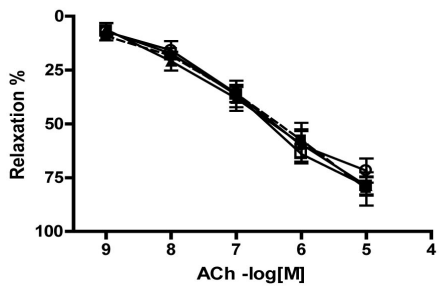

(d)

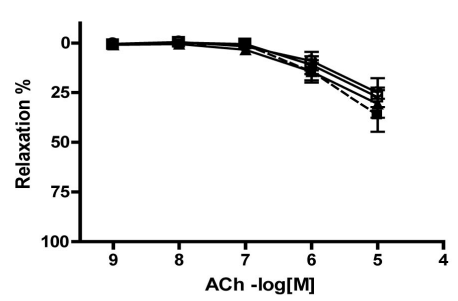

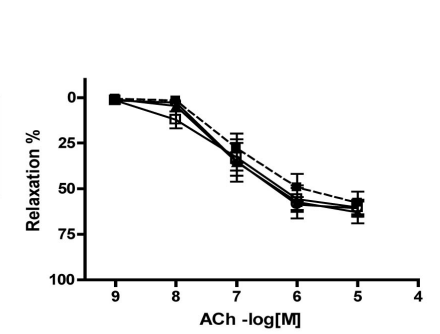

Aorta

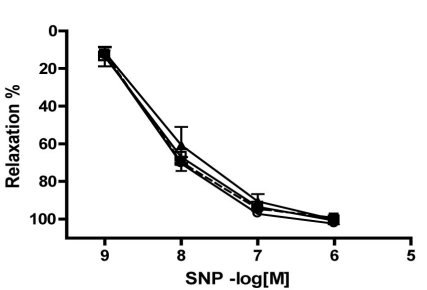

Nifedipine incubation

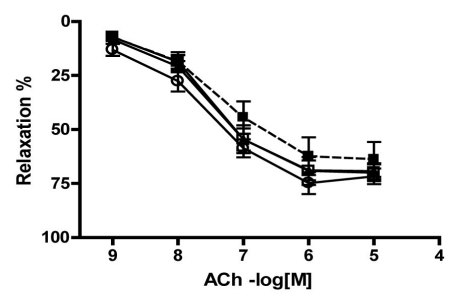

H-C vs other $\mathrm{p}<0.05$
W-C vs K-1, MK-7 $\mathrm{p}<0.05$

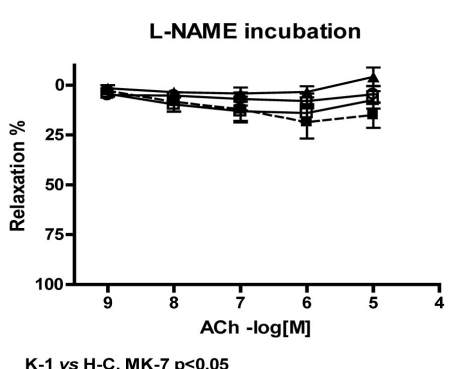

Figure 4. Acetylcholine-induced (ACh) (endothelium-dependent) (a) and sodium nitropruside-induced (SNP) (endothelium-independent) (b) relaxation after phenylephrine (PE) contraction of mesenteric artery (left column) and aorta (right column) rings. The role of L-type calcium channels (c) and nitric oxide (d) was studied using inhibitors (nifedipine and L-NAME, respectively). $\mathrm{H}-\mathrm{C}=$ Healthy control, $\mathrm{K}-1=$ Vitamin K-1, MK-7 = Menaquinone-7 and $\mathrm{W}-\mathrm{C}=$ Warfarin control. Mean \pm SEM, $\mathrm{n}=7-10$.

Kossa stainings. For some reason, systemic vascular calcification formed only in two warfarin-treated samples. It seems that the warfarin model did not work as expected based on the results from two histological staining methods for calcium detection: Alizarin Red S and von Kossa (Data not shown, $n=6$ - 8 in each group).

\subsection{Clinical Chemistry}

The results from serum clinical chemistry analysis are shown in Table 3. The levels of triglycerides, glucose and 
urea were reduced and the creatinine concentration was increased in the all warfarin-treated groups compared to the healthy controls. Alkaline phosphatase levels were decreased in the K-1 group vs. the healthy and warfarin control groups. Low density lipopolysaccharide (LDL) was decreased and total cholesterol increased in the healthy group. There were no differences in high density lipopolysaccharide (HDL) levels between the groups.

Clinical chemistry analysis from urine samples related to creatinine values is shown in Table 4. There were no significant differences between the groups, except urea levels were slightly higher in the warfarin-treated groups $(p<0.05)$. In addition, glucose and albumin excretions were somewhat elevated but not statistically significantly in the warfarin groups. Urine samples were collected with bladder puncture after sacrifice and therefore are not as reliable as from $24 \mathrm{~h}$ collection.

\section{Discussion}

In the present study we have investigated the blood pressure and vascular effects of vitamin K-1 and menaquinone-7 in an animal model of vascular calcification induced by warfarin in young Wistar-Kyoto rats. We used

Table 3. Clinical chemistry parameters from serum samples. Mean \pm SEM, $n=9-10$.

\begin{tabular}{|c|c|c|c|c|}
\hline & Healthy control & Vitamin K-1 group & Menaquinone-7 group & Warfarin control \\
\hline Sodium (mmol/l) & $139.4 \pm 0.3$ & $138.6 \pm 0.3$ & $138.8 \pm 0.6$ & $138.8 \pm 0.3$ \\
\hline Potassium (mmol/l) & $5.1 \pm 0.2$ & $5.0 \pm 0.2$ & $4.9 \pm 0.2$ & $5.0 \pm 0.2$ \\
\hline Calcium (mmol/l) & $2.77 \pm 0.04$ & $2.70 \pm 0.04$ & $2.66 \pm 0.03$ & $2.72 \pm 0.06$ \\
\hline Chloride (mmol/l) & $97.0 \pm 0.3$ & $97.4 \pm 0.3$ & $97.8 \pm 0.4$ & $97.2 \pm 0.2$ \\
\hline Inorganic phosphate (mmol/l) & $1.98 \pm 0.08$ & $1.92 \pm 0.06$ & $1.88 \pm 0.03$ & $1.94 \pm 0.06$ \\
\hline Glucose (mmol/l) & $9.95 \pm 0.09 \mathrm{f}$ & $8.86 \pm 0.13$ & $9.07 \pm 0.17$ & $9.12 \pm 0.08$ \\
\hline Cholesterol (mmol/l) & $2.05 \pm 0.05 \times$ & $1.87 \pm 0.04$ & $1.99 \pm 0.04$ & $1.95 \pm 0.05$ \\
\hline HDL (mmol/l) & $0.669 \pm 0.02$ & $0.661 \pm 0.016$ & $0.716 \pm 0.012$ & $0.721 \pm 0.013$ \\
\hline LDL (mmol/l) & $0.188 \pm 0.004 \bullet$ & $0.217 \pm 0.008$ & $0.259 \pm 0.021$ & $0.224 \pm 0.008$ \\
\hline Triglycerides (mmol/l) & $2.7 \pm 0.23 \mathrm{I}$ & $1.19 \pm 0.12$ & $1.17 \pm 0.06$ & $1.36 \pm 0.08$ \\
\hline Total protein (g/l) & $59.3 \pm 0.4$ & $59.1 \pm 0.3$ & $59.3 \pm 0.3$ & $59.5 \pm 0.4$ \\
\hline Albumin (g/l) & $38.0 \pm 0.4$ & $37.3 \pm 0.2$ & $37.7 \pm 0.4$ & $37.8 \pm 0.2$ \\
\hline Alanine amino transferase (U/l) & $22.2 \pm 1.2$ & $23.9 \pm 1.2$ & $25.3 \pm 1.8$ & $25.7 \pm 1.3$ \\
\hline Alkaline phosphatase (U/l) & $322 \pm 8$ & $277 \pm 15^{*}$ & $310 \pm 12$ & $340 \pm 6$ \\
\hline Aspartate amino transferase (U/l) & $55.2 \pm 4.8$ & $51 \pm 4$ & $44.6 \pm 1.4$ & $43.7 \pm 1.2$ \\
\hline Creatine kinase (U/l) & $198 \pm 43$ & $192 \pm 47$ & $140 \pm 15$ & $132 \pm 12$ \\
\hline Creatinine $(\mu \mathrm{mol} / \mathrm{l})$ & $43.0 \pm 0.6 \mathrm{I}$ & $47.6 \pm 0.7$ & $47.3 \pm 1.0$ & $48.3 \pm 0.5$ \\
\hline Urea (mmol/l) & $6.58 \pm 0.11 \mathrm{f}$ & $5.97 \pm 0.14$ & $6.01 \pm 0.12$ & $6.22 \pm 0.17$ \\
\hline
\end{tabular}

"K-1 vs. H-C and W-C p < 0.05; $\mathrm{f}$ H-C vs. all other groups $\mathrm{p}<0.05$; $\bullet \mathrm{H}-\mathrm{C}$ vs. MK-7 $\mathrm{p}<0.01 ; \times$ H-C vs. K-1 $\mathrm{p}<0.01$.

Table 4. Clinical chemistry parameters from urine samples. Mean \pm SEM, $n=9-10 . \times$ All parameters were related to creatinine values.

\begin{tabular}{|c|c|c|c|c|}
\hline & Healthy control & Vitamin K-1 group & Menaquinone-7 group & Warfarin control \\
\hline Sodium & $15.1 \pm 2.8$ & $17.5 \pm 4.2$ & $17.1 \pm 2.9$ & $14.4 \pm 2.3$ \\
\hline Potassium & $11.3 \pm 1.1$ & $11.7 \pm 1.2$ & $8.1 \pm 1.5$ & $10.0 \pm 1.7$ \\
\hline Calcium & $0.216 \pm 0.03$ & $0.207 \pm 0.019$ & $0.279 \pm 0.067$ & $0.319 \pm 0.084$ \\
\hline Chloride & $10.9 \pm 1.3$ & $15.4 \pm 4.2$ & $11.3 \pm 1.7$ & $10.7 \pm 1.8$ \\
\hline Inorganic phosphate & $0.671 \pm 0.157$ & $0.426 \pm 0.165$ & $0.332 \pm 0.113$ & $0.518 \pm 0.114$ \\
\hline Glucose & $0.125 \pm 0.005$ & $0.147 \pm 0.008$ & $0.141 \pm 0.007$ & $0.152 \pm 0.008$ \\
\hline Total protein & $674 \pm 111$ & $907 \pm 270$ & $765 \pm 175$ & $777 \pm 251$ \\
\hline Albumin & $1.66 \pm 0.25$ & $2.32 \pm 0.51$ & $2.08 \pm 0.42$ & $2.12 \pm 0.37$ \\
\hline Urea & $112,7 \pm 3.5^{*}$ & $122.0 \pm 3.6$ & $120.7 \pm 3.6$ & $132.6 \pm 4.8$ \\
\hline Creatinine $\times(\mu \mathrm{mol} / \mathrm{l})$ & $8771 \pm 979$ & $7399 \pm 1071$ & $7444 \pm 862$ & $6672 \pm 818$ \\
\hline
\end{tabular}

*Healthy control (H-C) vs. Warfarin control (W-C) p $<0.05$. 
four different treatment groups (healthy control, warfarin control, vitamin K-1, and menaquinone-7). All the tested compounds were administered mixed in the feed. During the five-week study-period, blood pressure was recorded weekly and at the end of the experiment, vascular function studies were performed using aortic and mesenteric artery segments in vitro.

The formation of vascular calcification was not detected systematically in all the warfarin-treated groups although we adapted our experimental design, doses, diets, and duration of the experiment, from earlier studies [5]-[8] with a similar study hypothesis. Howe et al. [6] used younger rats of different strain (Sprague-Dawley), and K-1 and warfarin dosage were administered subcutaneously which makes it difficult to compare the results and doses to this investigation. However, in the study by Spronk et al., [7] the design was highly similar. Previous studies have shown that vitamin K-2 [5] and menaquinone-4 [7], a shorter menaquinone derivative than MK-7, is able to prevent warfarin-induced vascular calcification and K-1 (doses between $1-6 \mathrm{mg} / \mathrm{g}$ food) had no effect on the formation of calcium deposits [7]. However, in study by Spronk et al. [7] menaquinone dose was 15-fold higher (1.5 mg/g feed) than in our experiment and Schungers et al. [8] provided no detailed data regarding which form of menaquinone their vitamin K-2 preparation contained, though the used dose $(100 \mathrm{\mu g} / \mathrm{g}$ food) was the same as in our study.

Over the five-week study-period systolic and diastolic blood pressure remained unchanged between the treatment groups. Heart rates also showed no difference between the groups. Recent studies [9]-[12] have shown that warfarin treatment increases systolic blood pressure of rats. However, warfarin treatment in our study failed to increase systolic blood pressure probably due to poor vascular calcification, and it is possible that mild calcification does not affect vascular stiffness and function enough to influence blood pressure. This assumption is also supported by the results from the present vascular reactivity studies, which showed that warfarin treatment influenced neither vascular endothelium dependent nor independent relaxation of the aorta (large vessel) nor that of the mesenteric arteries (small vessel). This is in agreement with the findings of Essalihi et al. [17] in which only aortic responses were tested. After calcium channel blockade using nifedipine, all warfarin-treated groups showed slightly better endothelium-dependent relaxation $(\mathrm{p}<0.05)$ in the aorta and after nitric oxide synthase (NOS) inhibition using L-NAME, endothelium-dependent relaxation were slightly better in healthy controls, and menaquinone-7 groups $(\mathrm{p}<0.05)$. This phenomenon was not seen in the mesenteric arteries. We also tried to measure calcium concentrations of the aorta segments. The method (Inductively coupled plasma mass spectrometry, ICP-MS) was not sensitive enough for the small concentrations of calcium (data not shown).

Interestingly, in the mesenteric artery rings, warfarin treatment reduced contractibility of the vessels reducing it especially after $\mathrm{KCl}$ contraction, and slightly after PE stimulation. This was not seen in aortas suggesting that warfarin treatment can modify contractibility in smaller vessels independently of possible calcification. However, endothelium-dependent relaxation was slightly impaired in this group. This could be associated to smaller contraction.

Warfarin treatment augmented the weight gain of the rats, which could not be explained by increased feed consumption. It also affected fat metabolism by decreasing total cholesterol and increasing LDL and triglycerides in serum, however, there were no differences between the warfarin-treated groups. This phenomenon may have something to do with weight development. Warfarin treatment also slightly influenced kidney function seen in urine clinical chemistry values of which urea and albumin levels were elevated, though, the kidney size was smaller (related to body mass) in H-C groups compared to the warfarin-treated groups, although this may be due to the higher body mass of $\mathrm{H}-\mathrm{C}$ group.

Alkaline phosphatase levels were decreased in the K-1 group vs. H-C and W-C groups. In the MK-7 group, alkaline phosphatase was also slightly reduced. Alkaline phosphatase promotes calcification by reducing pyrophosphate levels. Pyrophosphate is a potent inhibitor of medial vascular calcification in vitro and in vivo [18] [19]. Decreased alkaline phosphatase levels may thus have a vascular calcification protecting property. In addition, serum glucose and urea were increased and creatinine was decreased in H-C group compared to the warfarin-treated groups. Although, all clinical chemistry values were in normal range [20], thus severe pathological conditions can be left out of the discussion.

Clinically the question on possible role of vitamin K-1 or K-2 deficiency and its relation to coronary artery and other arterial calcifications under warfarin therapy is often forgotten or neglected. The deficiency may be more evident e.g. in vasculature than in the liver [21]. Vitamin K-1 originates mainly from green vegetables in the diet but microbes in the gut are also important K-vitamin producers seen as unbalanced vitamin K-status during chronic treatment with broad spectrum antibiotics. A short comprehensive review has recently been pub- 
lished in connection of a case report of arterial calcification associated with warfarin induced or dietary K-vitamin deficiency [22]. We have made a pilot evaluation from existing clinical laboratory data trying to find out correlation between blood INR values and serum calcium levels, but were not able to find it (Helin et al. unpublished). Further clinical or epidemiological studies are needed to solve relations between vitamin K-deficiency, warfarin therapy and arterial calcification.

\section{Conclusion}

Warfarin alone or together with vitamin $\mathrm{K}$ derivatives did not affect either blood pressure or endothelium-dependent or -independent relaxation of rat mesenteric arteries and aortas. Moreover, it did not induce systematic calcification of elastic lamellaes of rat aorta. However, warfarin treatment had significant effects on body weight and clinical chemistry parameters. It also slightly increased the endothelium-dependent relaxation of aorta after the L-type calcium channels were blocked. The vascular relaxation improved after NOS inhibition in healthy controls and MK-7 treated animals, indicating that in those groups the relaxation was not totally dependent on nitric oxide. Our results do not support previous observations on warfarin-induced vascular calcification and the role of vitamin $\mathrm{K}$.

\section{Acknowledgements}

We are grateful to Jouko Levijoki, Orion Oy for clinical chemistry measurements, Matti Airaksinen, professor of anatomy and Dr. Mikko Mäyränpää for their skilled help with histological samples evaluations, Inkeri Jänis, MetropoliLab for ICP-MS measurements and Dr. Mona Møller for her scientific contribution to the study protocol. The study was partly supported by DuPont Nutrition and Health, Finska Läkaresällskapet, and Finnish Clinical Chemistry Foundation. All the authors read and approved the final manuscript.

\section{Conflict of Interest}

Kirsti Tiihonen, Kaisa Olli and Krista Salli are employees of DuPont Nutrition and Health research center. The authors declare that there is no conflict of interest regarding the publication of this article.

\section{References}

[1] Kalra, S.S. and Shanahan, C.M. (2012) Vascular Calcification and Hypertension: Cause and Effect. Annals of Medicine, 44, S85-S92. http://dx.doi.org/10.3109/07853890.2012.660498

[2] Geleijnse, J.M., Vermeer, C., Grobbee, D.E., Schurgers, L.J., Knapen, M.H.J., van der Meer, I.M., et al. (2004) Dietary Intake of Menaquinone Is Associated with a Reduced Risk of Coronary Heart Disease: The Rotterdam Study. Journal of Nutrition, 134, 3100-3105.

[3] Beulens, J.W.J., Bots, M.L., Atsma, F., Bartelink, M.-A.E.L., Prokop, M., Geleijnse, J.M., et al. (2008) High Dietary Menaquinone Intake Is Associated with Reduced Coronary Calcification. Atherosclerosis, 203, 489-493. http://dx.doi.org/10.1016/j.atherosclerosis.2008.07.010

[4] Gast, G.C.M., de Roos, N.M., Bots, M.L., Beulens, J.W.J., Geleijnse, J.M., Witteman, J.C., et al. (2009) High Menaquinone Intake Reduces the Incidence of Coronary Heart Disease. Nutrition, Metabolism and Cardiovascular Diseases, 19, 504-507. http://dx.doi.org/10.1016/j.numecd.2008.10.004

[5] Price, P.A., Faus, S.A. and Williamson, M.K. (1998) Warfarin Causes Rapid Calcification of the Elastic Lamellae in Rat Arteries Heart Valves. Arteriosclerosis, Thrombosis, and Vascular Biology, 18, 1400-1407. http://dx.doi.org/10.1161/01.ATV.18.9.1400

[6] Howe, A.M. and Webster, W.S. (2000) Warfarin Exposure and Calcification of the Arterial System of the Rat. International Journal of Experimental Pathology, 81, 51-56. http://dx.doi.org/10.1046/j.1365-2613.2000.00140.x

[7] Spronk, H.M.H., Soute, B.A.M., Schungers, L.J., Thijssen, H.H.W., DeMey, J.G.R. and Vermeer, C. (2003) TissueSpecific Utilization of Menaquinone-4 Results in the Prevention of Arterial Calcification in Warfarin-Treated Rats. Journal of Vascular Research, 40, 531-537. http://dx.doi.org/10.1159/000075344

[8] Schungers, L.J., Spronk, H.M.H., Soute, B.A.M., Schiffers, P.M., DeMey, J.G.R. and Vermeer, C. (2007) Regression of Warfarin-Induced Medial Elastocalcinosis by High Inkake of Vitamin K in Rats. Blood, 109, 2823-2831.

[9] Dao, H.H., Essalihi, R., Graillon, J.-F., Lariviere, R., de Champlain, J. and Moreau, P. (2002) Pharmacological Prevention and Regression of Arterial Remodeling in a Rat Model of Isolated Systolic Hypertension. Journal of Hypertension, 20, 1597-1606. http://dx.doi.org/10.1097/00004872-200208000-00023 
[10] Essalihi, R., Zandvliet, M.L., Moreau, S., Gilbert, L.-A., Bouvet, C., Lenoel, C., et al. (2007) Distinct Effects of Amlopidine Treatment on Vascular Elastocalcinosis and Stiffness in a Rat Model of Isolated Systolic Hypertension. Journal of Hypertension, 25, 1879-1886. http://dx.doi.org/10.1097/HJH.0b013e328255e906

[11] Liu, C.Y., Wan, J.J., Yang, Q.F., Qi, B.L., Peng, W. and Chen, X.L. (2008) Effects of Atorvastatin on WarfarinInduced Aortic Medial Calcification and Systolic Blood Pressure in Rats. Journal of Huazhong University of Science and Technology, 28, 535-538. http://dx.doi.org/10.1007/s11596-008-0510-1

[12] Beazley, K.E., Banyard, D., Lima, F., Deasey, S.C., Nurminsky, D.I., Komoplyannikov, M. and Nurminskaya, M.V. (2013) Transglutaminase Inhibitors Attenuate Vascular Calcification in a Preclinical Model. Arteriosclerosis, Thrombosis, and Vascular Biology, 33, 43-51. http://dx.doi.org/10.1161/ATVBAHA.112.300260

[13] Ferland, G. (1998) The Vitamin K-Dependent Proteins: An Update. Nutrition Reviews, 56, 223-230. http://dx.doi.org/10.1111/j.1753-4887.1998.tb01753.x

[14] Luo, G., Ducy, P., McKee, M.G., Pinero, G.J., Loyer, E., Behringer, R.R. and Karsenty, G. (1998) Spontaneous Calcification of Arteries and Cartilage in Mice Lacking Matrix GLA Protein. Nature, 386, 78-81. http://dx.doi.org/10.1038/386078a0

[15] Danziger, J. (2008) Vitamin K-Dependent Proteins, Warfarin, and Vascular Calcification. Clinical Journal of the American Society of Nephrology, 3, 1504-1510. http://dx.doi.org/10.2215/CJN.00770208

[16] Erkkilä, A.T. and Booth, S.L. (2008) Vitamin K Intake and Atherosclerosis. Current Opinion in Lipidology, 19, 39-42. http://dx.doi.org/10.1097/MOL.0b013e3282f1c57f

[17] Essalihi, R., Dao, H.H., Yamaguchi, N. and Moreau, P. (2003) A New Model of Isolated Systolic Hypertension Induced by Chronic Warfarin and Vitamin K1 Treatment. American Journal of Hypertension, 16, 103-110. http://dx.doi.org/10.1016/S0895-7061(02)03204-1

[18] Lomashvili, K.A., Cobbs, S., Hennigar, R.A., Hardcastle, K.I. and O’Neill, W.C. (2004) Phosphate-Induced Vascular Calcification: Role of Pyrophosphate and Osteopontin. Journal of the American Society of Nephrology, 15, 1392-1401. http://dx.doi.org/10.1097/01.ASN.0000128955.83129.9C

[19] Schibler, D., Russell, G.G. and Fleisch, H. (1968) Inhibition by Pyrophosphate and Polyphosphate of Aortic Calcification Induced by Vitamin D3 in Rats. Clinical Science, 35, 363-372.

[20] Boehm, O., Zu, B., Koch, A., Tran, N., Freyenhagen, R., Hartmann, M. and Zacharowski, K. (2007) Clinical Chemistry Reference Database for Wistar Rats and C57/BL6 Mice. Biological Chemistry, 388, 547-554. http://dx.doi.org/10.1515/BC.2007.061

[21] Zhu, D., Mackenzie, N.C., Farquhaarson, C. and Macrae, V.E. (2012) Mechanisms and Clinical Consequences of Vascular Calcification. Frontiers in Endocrinology (Lausanne), 3, 95. http://dx.doi.org/10.3389/fendo.2012.00095

[22] Wahlqvist, M.L., Tanaka, K. and Tzeng, B.H. (2013) Clinical Decision-Making for Vitamin K-1 and K-2 Deficiency and Coronary Artery Calcification with Warfarin Therapy: Are Diet, Factor Xa Inhibitors or Both the Answer? Asia Pacific Journal of Clinical Nutrition, 22, 492-496. 
Scientific Research Publishing (SCIRP) is one of the largest Open Access journal publishers. It is currently publishing more than 200 open access, online, peer-reviewed journals covering a wide range of academic disciplines. SCIRP serves the worldwide academic communities and contributes to the progress and application of science with its publication.

Other selected journals from SCIRP are listed as below. Submit your manuscript to us via either submit@scirp.org or Online Submission Portal.
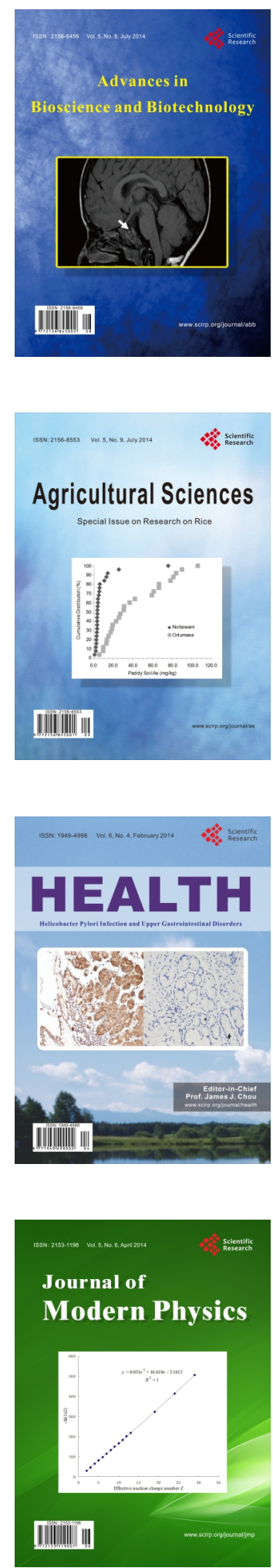
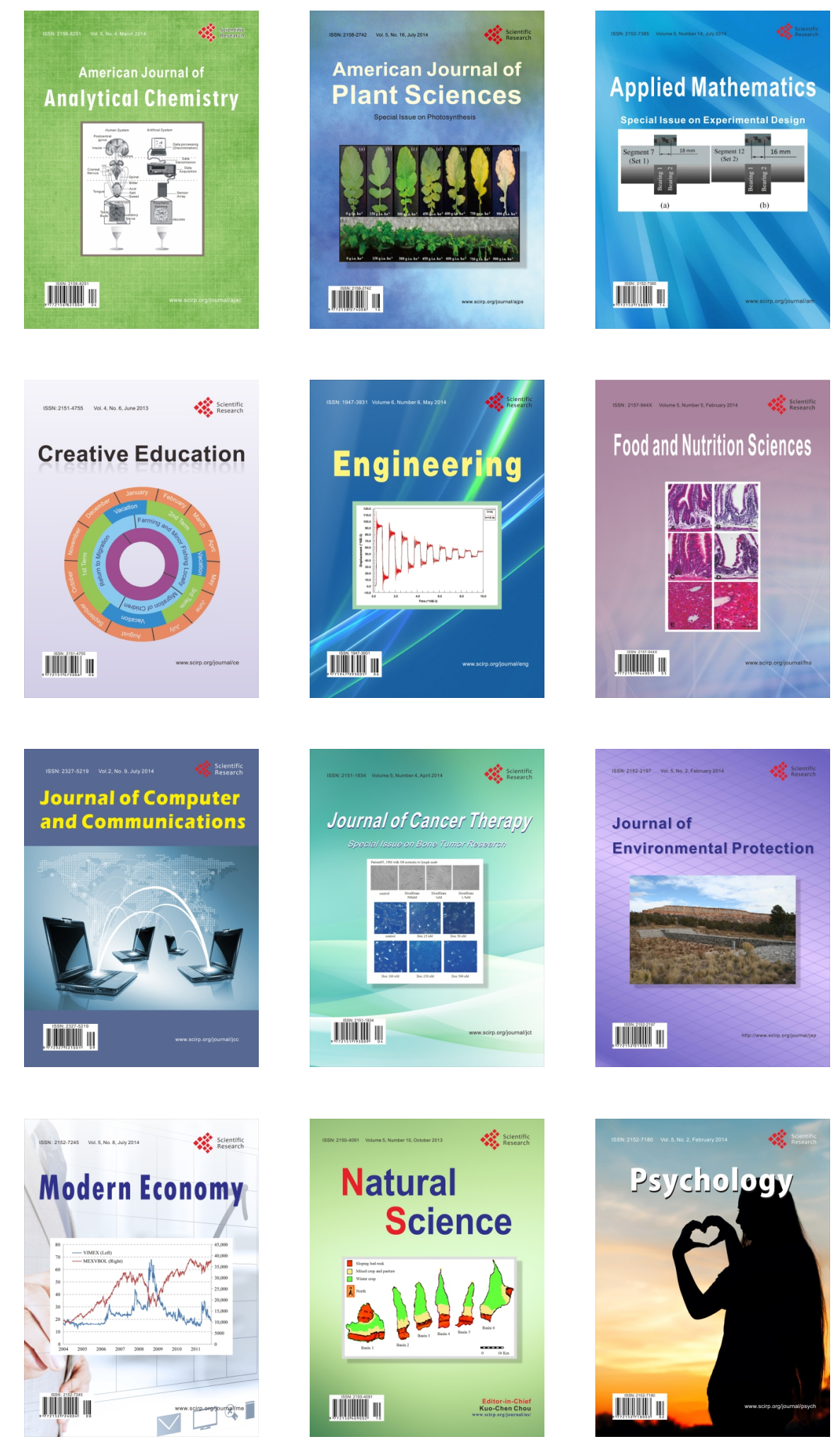